\title{
Reflexive Scott domains are not complete for the extensional lambda calculus
}

\author{
Alberto Carraro ${ }^{\circ \dagger}$ \\ † Laboratoire PPS \\ Université Paris Diderot \\ Paris, France \\ Email: acarraro@dsi.unive.it
}

\author{
Antonino Salibra ${ }^{\circ}$ \\ - Dipartimento di Informatica \\ Università Ca' Foscari di Venezia \\ Venezia, Italia \\ Email: salibra@dsi.unive.it
}

\begin{abstract}
A longstanding open problem is whether there exists a model of the untyped $\lambda$-calculus in the category $\mathrm{Cpo}$ of complete partial orderings and Scott continuous functions, whose theory is exactly the least $\lambda$-theory $\lambda \beta$ or the least extensional $\lambda$-theory $\lambda \beta \eta$. In this paper we analyze the class of reflexive Scott domains, the models of $\lambda$-calculus living in the category of Scott domains (a full subcategory of Cpo). The following are the main results of the paper:

(i) Extensional reflexive Scott domains are not complete for the $\lambda \beta \eta$-calculus, i.e., there are equations not in $\lambda \beta \eta$ which hold in all extensional reflexive Scott domains.

(ii) The order theory of an extensional reflexive Scott domain is never recursively enumerable.

These results have been obtained by isolating among the reflexive Scott domains a class of webbed models arising from Scott's information systems, called iweb-models. The class of iweb-models includes all extensional reflexive Scott domains, all preordered coherent models and all filter models living in Cpo. Based on a fine-grained study of an "effective" version of Scott's information systems, we have shown that there are equations not in $\lambda \beta$ (resp. $\lambda \beta \eta$ ) which hold in all (extensional) iweb-models.
\end{abstract}

Keywords-Lambda calculus, lambda theories, Reflexive Scott domains, Filter models.

\section{INTRODUCTION}

Lambda theories are congruences on the set of $\lambda$-terms, which contain $\beta$-conversion; extensional $\lambda$-theories are those which contain $\beta \eta$-conversion. Lambda theories arise by syntactical or by semantic considerations. Indeed, a $\lambda$-theory may correspond to a possible operational (observational) semantics of $\lambda$-calculus, as well as it may be induced by a model of $\lambda$-calculus through the kernel congruence relation of the interpretation function. Although researchers have, till recently, mainly focused their interest on a limited number of them, the lattice of $\lambda$-theories ordered by inclusion constitutes a very rich, interesting and complex mathematical structure of cardinality $2^{\aleph_{0}}$ (see [1], [3], [4]).

After the first model, found by Scott in 1969 in the category of complete lattices and Scott continuous functions, a large number of mathematical models for $\lambda$-calculus, arising from syntax-free constructions, have been introduced in various Cartesian closed categories (ccc, for short) of domains and were classified into semantics according to the nature of their representable functions, see e.g. [1], [3],
[17]. Scott continuous semantics [19] is the class of reflexive cpo-models, that are reflexive objects in the category $\mathbf{C p o}$ whose objects are complete partial orders and morphisms are Scott continuous functions. The stable semantics (Berry [6]) and the strongly stable semantics (Bucciarelli-Ehrhard [7]) are refinements of the continuous semantics, introduced to approximate the notion of "sequential" Scott continuous function; finally "weakly continuous" semantics have been introduced, either for modeling non determinism, or for foundational purposes [3], [11]. In each of these semantics all models come equipped with a partial order, and some of them, called webbed models, are built from lower level structures called "webs". The simplest class of webbed models is the class of graph models, which was isolated in the seventies by Plotkin, Scott and Engeler within the continuous semantics. The class of graph models contains the simplest models of $\lambda$-calculus, is itself the easiest describable class, and represents nevertheless $2^{\aleph_{0}}$ (non-extensional) $\lambda$-theories. Another example of a class of webbed models, and the most established one, is the class of filter models. It was isolated at the beginning of the eighties by Barendregt, Dezani and Coppo [2], after the introduction of the intersection type discipline by Coppo and Dezani [9].

Scott continuous semantics and the other mentioned semantics are structurally and equationally rich. Ten years ago, Kerth [15], [16] has proved that in each of the above semantics it is possible to build up $2^{\aleph_{0}}$ models inducing pairwise distinct $\lambda$-theories. Nevertheless, the above denotational semantics do not match all possible operational semantics of $\lambda$-calculus. Honsell and Ronchi della Rocca [14] have shown that there exist theories which do not have models in the category Cpo. More recently, it has been proved in an uniform way that there are $2^{\aleph_{0}}$ theories which are omitted by all ordered models of $\lambda$-calculus with a bottom element [18].

The question of the existence of a non-syntactical model of $\lambda \beta$ (or $\lambda \beta \eta$, the least extensional $\lambda$-theory) has been circulating since at least the beginning of the eighties ${ }^{1}$, but it was only first raised in print in [14]. This problem is still open, but generated a wealth of interesting research and

\footnotetext{
${ }^{1}$ See Problem 22 in the list of TLCA open problems [13]
} 
results (surveyed in [3] and [4]), from which we only sketch below what is relevant for the present paper.

In 1995 Di Gianantonio, Honsell and Plotkin succeeded to build an extensional model having theory $\lambda \beta \eta$, living in some weakly continuous semantics [11]. However, the construction of this model as an inverse limit starts from the term model of $\lambda \beta \eta$, and hence involves the syntax of $\lambda$-calculus. Furthermore the existence of a model living in Scott semantics itself, or in one of its two refinements, remains completely open. Nevertheless, the authors also proved in [11] that the set of extensional theories representable by models living in Scott continuous semantics has a least element.

In view of the second result of [11], it becomes natural to ask whether, given a (uniformly presented) class of models of $\lambda$-calculus, there is a minimum $\lambda$-theory represented in it; a question which was raised in [3]. In [8] Bucciarelli and Salibra showed that the answer is also positive for the class of graph models, and that the least $\lambda$-theory in this class is different from $\lambda \beta$ and of course $\lambda \beta \eta$.

We notice also that there are only very few theories of non syntactical models which are known to admit an alternative (i.e., non-model-thoretic) description (e.g. via syntactical considerations), and that all happen to coincide either with the theory of Böhm trees [1] or some variations of it, and hence are not recursively enumerable (r.e., for short). This led Berline, Manzonetto and Salibra [5] to raise the following problem, which is a natural extension of the initial problem: can a model living in Scott continuous semantics or in one of its refinements have an r.e. equational theory? Based on a notion of an effective model of $\lambda$-calculus, in [5] it was shown that the order theory of an effective model cannot be r.e. and its equational theory is different from $\lambda \beta(\lambda \beta \eta)$. Effective models are omni-present: in particular, all the models which have been introduced individually in the literature can easily be proved effective. Concerning the above mentioned semantics, it was also proved that no effective model living in the stable or strongly stable semantics has an r.e. equational theory and that no order theory of a graph model can be r.e.

The category of algebraic cpos (a full subcategory of Cpo) has many nice properties but unfortunately lacks the essential characteristic of being a ccc: the function space of two algebraic cpos need not be algebraic. As the functionspace construction is often used in denotational semantics and the models of $\lambda$-calculus are reflexive objects in a ccc, this is a serious drawback. Fortunately there are Cartesian closed (full) subcategories of algebraic cpos. The most important, introduced by Scott [20], is the ccc SD of Scott domains (i.e., bounded complete algebraic cpos). Most of the reflexive cpo-models of $\lambda$-calculus introduced in the literature in the last forty years are reflexive Scott domains, i.e., reflexive objects in SD.

In this paper we work with a category equivalent to SD but more "concrete" and easier to work with, namely the ccc Inf of information systems introduced by Scott in early eighties [20]. We use Inf to isolate a class of webbed models, called iweb-models, which is strictly contained within the class of reflexive Scott domains, but includes all extensional reflexive Scott domains, all preordered coherent models and all filter models living in Cpo. Based on a finegrained study of an "effective" version of Scott's information systems, in the key technical theorem of this paper we prove that there exists an effective model of $\lambda$-calculus, not living in Cpo, whose order theory is contained within the order theory of every iweb-model. By applying the above mentioned results on effective models [5] and Selinger's result [21] on the triviality of every compatible order on the term model of $\lambda \beta(\lambda \beta \eta)$, we show that the order theory of an iweb-model is never r.e., and that there are equations not in $\lambda \beta$ (resp. $\lambda \beta \eta$ ) which hold in all (extensional) iwebmodels. As a consequence we get the main results of the paper:

(i) Extensional reflexive Scott domains are not complete for the $\lambda \beta \eta$-calculus, i.e., there are equations not in $\lambda \beta \eta$ which hold in all extensional reflexive Scott domains.

(ii) The order theory of an extensional reflexive Scott domain is never r.e.

\section{Preliminaries}

If $A$ is a set, then we denote by $\mathcal{P}(A)$ the power set of $A$ and by $\mathcal{P}_{\mathrm{f}}(A)$ the set of all finite subsets of $A$. We write $a \subseteq_{\mathrm{f}} A$ for $a \subseteq A$ and $a$ is finite.

If $f: A \rightarrow B$ is a function and $Y \subseteq A$, then $f(Y)=$ $\{f(y): y \in Y\}$.

\section{A. Partial Orderings}

Let $\left(\mathcal{D}, \sqsubseteq_{\mathcal{D}}\right)$ be a partially ordered set (poset, for short). When there is no ambiguity we write $\mathcal{D}$ instead of $\left(\mathcal{D}\right.$, $\left.\subseteq_{\mathcal{D}}\right)$. Two elements $u$ and $v$ of $\mathcal{D}$ are: comparable if either $u \sqsubseteq \mathcal{D} v$ or $v \sqsubseteq_{\mathcal{D}} u . A \subseteq \mathcal{D}$ is directed if, for all $u, v \in A$, there exists $z \in A$ such that $u \sqsubseteq \mathcal{D} z$ and $v \sqsubseteq \mathcal{D} z . A \subseteq \mathcal{D}$ is downward closed if $v \in A$ and $u \sqsubseteq \mathcal{D} v$ imply $u \in A$.

A poset $\mathcal{D}$ is a complete partial order (cpo, for short) if it has a least element (denoted by $\perp_{\mathcal{D}}$ ) and every directed set $A \subseteq \mathcal{D}$ admits a least upper bound (denoted by $\sqcup A$ ). If $\mathcal{D}$ is a cpo, then $[\mathcal{D} \rightarrow \mathcal{D}]$ denotes the cpo of Scott continuous functions ordered pointwise. Cpo denotes the Cartesian closed category ( $c c c$, for short) of cpos and Scott continuous functions.

An element $d \in \mathcal{D}$ is called compact if for every directed $A \subseteq \mathcal{D}$ we have that $d \sqsubseteq_{\mathcal{D}} \sqcup A$ implies $d \sqsubseteq_{\mathcal{D}} v$ for some $v \in$ $A$. We write $\mathcal{K}(\mathcal{D})$ for the collection of compact elements of $\mathcal{D}$. A cpo $\mathcal{D}$ is algebraic if for every $u \in \mathcal{D}$ the set $\left\{d \in \mathcal{K}(\mathcal{D}): d \sqsubseteq_{\mathcal{D}} u\right\}$ is directed and $u$ is its least upper bound.

A cpo is bounded complete if $\sqcup\{u, v\}$ exists for all upper bounded elements $u, v$. A bounded complete algebraic cpo 
is called a Scott domain [20]. The category SD of Scott domains and Scott continuous functions is a ccc.

\section{B. Numberings}

We denote by $\mathbb{N}$ the set of natural numbers. A set $A \subseteq \mathbb{N}$ is r.e. if it is the domain of a partial recursive function. The complement of a r.e. set is called a co-r.e. set. If both $A$ and its complement are r.e., $A$ is called decidable or recursive.

Definition 1. A numeration is a pair $(A, \sigma)$, where $\sigma$ : $\mathbb{N} \rightarrow A$ is a surjective total map; the function $\sigma$ is called a numbering of $A$. We write $\sigma_{n}$ in place of $\sigma(n)$.

Let $e: \mathcal{P}_{\mathrm{f}}(\mathbb{N}) \cong \mathbb{N}$ be the well known coding of finite sets of natural numbers. If $(A, \sigma)$ is a numeration, then the function $\widehat{\sigma}$, defined by $\widehat{\sigma}(n)=\left\{\sigma_{m}: m \in e^{-1}(n)\right\}$, is a numbering of $\mathcal{P}_{\mathrm{f}}(A)$. Let $\left\langle_{-},{ }_{-}\right\rangle: \mathbb{N} \times \mathbb{N} \cong \mathbb{N}$ be the well known coding of pairs of natural numbers. If $(A, \sigma)$ and $(B, \tau)$ are two numerations, then the function $\sigma \times \tau$, defined by $(\sigma \times \tau)(\langle n, m\rangle)=\left(\sigma_{n}, \tau_{m}\right)$, is a numbering of $A \times B$. Moreover, we say that:

- A subset $X \subseteq A$ is $\sigma$-ree. (decidable) if $\left\{n: \sigma_{n} \in X\right\}$ is r.e. (decidable).

- A partial function $f: A \rightarrow B$ is $(\sigma, \tau)$-computable if there exists a partial recursive function $\varphi: \mathbb{N} \rightarrow \mathbb{N}$ such that $\sigma_{n} \in \operatorname{dom}(f)$ iff $n \in \operatorname{dom}(\varphi)$, and $f\left(\sigma_{n}\right)=\tau_{\varphi(n)}$. In such a case, we say that $\varphi$ tracks $f$. If $\operatorname{dom}(\varphi)$ is decidable, then $f$ is called strongly computable.

Definition 2. A numeration $(A, \sigma)$ is computable iff the equality relation on $A$ is $\sigma$-decidable.

Note that if $(A, \sigma)$ and $(B, \tau)$ are computable, then so are $\left(\mathcal{P}_{\mathrm{f}}(A), \widehat{\sigma}\right)$ and $(A \times B, \sigma \times \tau)$.

\section{Lambda calculus and lambda models}

With regard to the $\lambda$-calculus we follow the notation and terminology of [1]. $\Lambda$ and $\Lambda^{\circ}$ are, respectively, the set of $\lambda$-terms and of closed $\lambda$-terms. We denote $\alpha \beta$-conversion by $\lambda \beta$. A $\lambda$-theory is a congruence on $\Lambda$ (with respect to the operators of abstraction and application) which contains $\lambda \beta$. A $\lambda$-theory is extensional if it contains the equation $\lambda x . x=\lambda x y . x y ; \lambda \beta \eta$ is the least extensional $\lambda$-theory.

If $\mathbf{C}$ is a category, then a pair $(F, G)$ is a retraction pair from an object $\mathcal{E}$ into an object $\mathcal{D}$ if $F: \mathcal{D} \rightarrow \mathcal{E}$ and $G: \mathcal{E} \rightarrow \mathcal{D}$ are two morphisms satisfying $F \circ G=i d_{\mathcal{E}}$. If the morphims in $\mathbf{C}$ are partially ordered, then a retraction pair satisfying $G \circ F \leq i d_{\mathcal{D}}\left(G \circ F=i d_{\mathcal{D}}\right)$ is called an e.p.(iso-) pair.

It is well known [1, Ch. 5] that a model of the untyped $\lambda$-calculus, or $\lambda$-model here, is nothing else than a reflexive object $(\mathcal{D}, F, G)$ of a ccc $\mathbf{C}$, that is to say $(F, G)$ is a retraction pair from $[\mathcal{D} \rightarrow \mathcal{D}]$ into $\mathcal{D}$, where $[\mathcal{D} \rightarrow \mathcal{D}]$ is the exponent object of C. A $\lambda$-model is extensional if $(F, G)$ is an iso-pair, while it is semi-extensional if $(F, G)$ is an e.p.-pair.
In the following we will be interested in the ccc Cpo (Scott continuous semantics). Reflexive objects in Cpo will be called reflexive cpo-models, while reflexive objects in the ccc SD of Scott domains will be called reflexive Scott domains.

Let $\mathbf{D}=(\mathcal{D}, F, G)$ be a $\lambda$-model. We let $\operatorname{Env}_{\mathcal{D}}$ be the set of environments with values in $\mathcal{D}$. For every $x \in \operatorname{Var}$ and $d \in \mathcal{D}$ we denote by $\rho[x:=d]$ the environment $\rho^{\prime}$ which coincides with $\rho$, except on $x$, where $\rho^{\prime}$ takes the value $d$. The interpretation $|M|: \operatorname{Env}_{\mathcal{D}} \rightarrow \mathcal{D}$ of a $\lambda$-term $M$ is defined by structural induction on $M$, as follows:

- $|x|_{\rho}^{\mathbf{D}}=\rho(x) ; \quad|M N|_{\rho}^{\mathbf{D}}=F\left(|M|_{\rho}^{\mathbf{D}}\right)\left(|N|_{\rho}^{\mathbf{D}}\right) ;$

- $|\lambda x . M|_{\rho}^{\mathbf{D}}=G\left(d \in \mathcal{D} \mapsto|M|_{\rho[x:=d]}^{\mathbf{D}}\right)$.

We write $|M|^{\mathbf{D}}$ for $|M|_{\rho}^{\mathbf{D}}$ if $M$ is a closed $\lambda$-term.

Each $\lambda$-model $\mathbf{D}$ induces a $\lambda$-theory, denoted here by $\mathrm{Eq}(\mathbf{D})$, and called the equational theory of $\mathbf{D}$. Thus, $M=N \in \mathrm{Eq}(\mathbf{D})$ if, and only if, $M$ and $N$ have the same interpretation in $\mathbf{D}$. A partially ordered $\lambda$-model $\mathbf{D}$ induces also an order theory $\operatorname{Ord}(\mathbf{D})=\{M \sqsubseteq N$ : $|M|_{\rho}^{\mathbf{D}} \sqsubseteq_{D}|N|_{\rho}^{\mathbf{D}}$ for all environments $\left.\rho\right\}$.

\section{Effective lambda models}

In this section we introduce the notion of an effective $\lambda$ model and we present from [5] the main properties of these models.

Definition 3. [12], [22] An effective Scott domain is a pair $(\mathcal{D}, \sigma)$ where $\mathcal{D}$ is a Scott domain, $(\mathcal{K}(\mathcal{D}), \sigma)$ is a numeration and the following predicates on $\mathcal{K}(\mathcal{D})$ are $\sigma$ decidable:

- $\operatorname{Com}=\left\{(x, y) \in \mathcal{K}(\mathcal{D})^{2}: \exists z \in \mathcal{K}(\mathcal{D}) . z \geq x, y\right\}$

- $\operatorname{Sup}=\left\{(x, y, z) \in \mathcal{K}(\mathcal{D})^{3}: z=x \sqcup y\right\}$.

It follows that the order relation $\sqsubseteq_{\mathcal{D}}$ and the equality on $\mathcal{K}(\mathcal{D})$ are decidable, i.e., $\sigma$ is a computable numbering of $\mathcal{K}(\mathcal{D})$.

An element $x \in \mathcal{D}$ is called r.e. (decidable) if the set $\{y \in \mathcal{K}(\mathcal{D}): y \leq x\}$ is r.e. (decidable). We write $\mathcal{D}^{\text {r.e. }}$ $\left(\mathcal{D}^{d e c}\right)$ to indicate the set of all r.e. (decidable) elements of $\mathcal{D}$. Clearly $\mathcal{K}(\mathcal{D}) \subseteq \mathcal{D}^{\text {dec }}$.

If $\mathcal{D}$ is an effective Scott domain, then $[\mathcal{D} \rightarrow \mathcal{D}]$ is an effective Scott domain too.

Using standard techniques of recursion theory it is possible to get in a uniform way a numeration $\sigma^{\text {r.e. }}: \mathbb{N} \rightarrow \mathcal{D}^{\text {r.e. }}$ which is adequate in the sense that the relation $\sigma_{k} \sqsubseteq_{D} \sigma_{n}^{\text {r.e. }}$ is r.e. in $(k, n)$ and the inclusion mapping $\iota: K(\mathcal{D}) \rightarrow \mathcal{D}^{\text {r.e. }}$ is computable w.r.t. $\sigma, \sigma^{\text {r.e. }}$.

Proposition 4. [22, Ch. 10, Prop. 3.7] Let $(\mathcal{D}, \sigma)$ and $(\mathcal{E}, \tau)$ be effective Scott domains. Then the following statements are equivalent:

(i) $f \in[\mathcal{D} \rightarrow \mathcal{E}]^{\text {r.e. }}$

(ii) $\{(x, y) \in \mathcal{K}(\mathcal{D}) \times \mathcal{K}(\mathcal{E}): y \sqsubseteq \mathcal{E} f(x)\}$ is r.e.

(iii) The restriction $f \uparrow: \mathcal{D}^{\text {r.e. }} \rightarrow \mathcal{E}^{\text {r.e. }}$ is $\left(\sigma^{\text {r.e. }}, \tau^{\text {r.e. }}\right)$ computable. 
Definition 5. [5, Def. 5] $A \lambda$-model $\mathbf{D}=(\mathcal{D}, F, G)$ is weakly effective if $\mathcal{D}$ is an effective Scott domain, $F \in$ $[\mathcal{D} \rightarrow[\mathcal{D} \rightarrow \mathcal{D}]]^{\text {r.e. }}$ and $G \in[[\mathcal{D} \rightarrow \mathcal{D}] \rightarrow \mathcal{D}]^{\text {r.e. }}$.

If $\mathcal{D}$ is an effective domain, then the set $E n v_{\mathcal{D}}$ of environments is an effective domain too. Let $\Lambda_{\perp}=\Lambda \cup\{\perp\}$ be the usual flat domain of $\lambda$-terms. The element $\perp$ is always interpreted as $\perp_{\mathcal{D}}$ in a reflexive Scott domain $\mathcal{D}$.

Proposition 6. [5, Prop. 1] If $\mathbf{D}$ is a weakly effective $\lambda$ model and $|-|^{\mathbf{D}}$ is the interpretation function of $\lambda$-terms, then $|-|^{\mathbf{D}} \in\left[\Lambda_{\perp} \times \text { Env }_{\mathcal{D}} \rightarrow \mathcal{D}\right]^{\text {r.e. }}$.

A set $X \subseteq \Lambda^{o}$ is $\beta$-closed if $M \in X$ and $M={ }_{\lambda \beta} N$ imply $N \in X$, for all $M, N \in \Lambda^{o}$. Similarly for $X \subseteq \Lambda$. 4].

The following theorem is a slight modification of $[5, \mathrm{Thm}$.

Theorem 7. Let $\mathbf{D}$ be a weakly effective $\lambda$-model for which there exists $M \in \Lambda^{o}$ such that $|M|^{\mathbf{D}} \in \mathcal{D}^{\text {dec }}$. Then we have:

(i) $\left\{N \in \Lambda^{o}:|N|^{\mathbf{D}} \sqsubseteq|M|^{\mathbf{D}}\right\}$ is a $\beta$-closed co-r.e. set.

(ii) $\operatorname{Ord}(\mathbf{D})$ is not r.e.

(iii) $E q(\mathbf{D}) \neq \lambda \beta, \lambda \beta \eta$. $\mathcal{D}^{\text {dec }}$

Proof: (i) follows from Prop. 6 and the fact that $|M|^{\mathbf{D}} \in$

(ii) is an immediate consequence of (i).

(iii) follows from (ii) and Selinger's result [21] on the triviality of every compatible order on the term model of $\lambda \beta(\lambda \beta \eta)$

\section{INFORMATION SYSTEMS}

Scott domains have an appealing and suggestive representation as information systems introduced by Dana Scott in [20]. An information system consists of a set of tokens, which are related by entailment and consistency relations. It determines a Scott domain with elements those sets of tokens which are consistent and closed with respect to the entailment relation; the ordering is just set inclusion. Vice versa a Scott domain defines an information system through its compact elements. In this section we review the basic notions on information systems.

Definition 8. $A n$ information system is a triple $\mathcal{A}=$ $\left(A, \operatorname{Con}_{A}, \vdash_{A}\right)$, where $\operatorname{Con}_{A} \subseteq \mathcal{P}_{\mathrm{f}}(A)$ is a downward closed family containing all singleton sets, and $\vdash_{A} \subseteq$ $\mathrm{Con}_{A} \times A$ satisfy the three axioms listed below. We adopt the following conventions: letters $\alpha, \beta, \gamma, \ldots$ are used for tokens, i.e., elements of $A$; letters $a, b, c, \ldots$ are used for elements of $\operatorname{Con}_{A}$; letters $x, y, z, \ldots$ are used for arbitrary elements of $\mathcal{P}(A)$.

(IS1) if $\alpha \in a$, then $a \vdash_{A} \alpha$

(IS2) if $\forall \beta \in b . a \vdash_{A} \beta$ and $b \vdash_{A} \alpha$, then $a \vdash_{A} \alpha$

(IS3) if $a \vdash_{A} \alpha$, then $a \cup\{\alpha\} \in \operatorname{Con}_{A}$

We say that $x \subseteq A$ is consistent if $\mathcal{P}_{\mathrm{f}}(x) \subseteq$ Con. If $x$ is consistent, then we define: (i) $\Downarrow_{A} x=\left\{\alpha: a \vdash_{A} \alpha, a \subseteq_{\mathrm{f}} x\right\}$; (ii) $x \vdash_{A} \alpha$ if $\alpha \in \Downarrow_{A} x$; (iii) $x \vdash_{A} y$ if $y \subseteq \Downarrow_{A} x$. We often write $\Downarrow x$ for $\Downarrow_{A} x$. When we write $x \vdash \alpha$ or $x \vdash y$ it is always implicitly assumed that $x$ is consistent. The notation $\alpha \dashv \beta$ means $\alpha \vdash \beta$ and $\beta \vdash \alpha$.

A consistent subset $x \subseteq A$ is a point of $\mathcal{A}$ if $\Downarrow_{x}=x$. The set of all points of $\mathcal{A}$, ordered by inclusion, is a Scott domain.

Given information systems $\mathcal{A}$ and $\mathcal{B}$, a relation $R \subseteq$ $\mathrm{Con}_{A} \times \operatorname{Con}_{B}$ is said to be approximable ( [20, Def. 5.1]) if $(\emptyset, \emptyset) \in R$, and for all $a, a^{\prime} \in \operatorname{Con}_{A}$ and all $b, b^{\prime}, c \in \operatorname{Con}_{B}$ :

1. if $(a, b),(a, c) \in R$, then $b \cup c \in \operatorname{Con}_{B}$ and $(a, b \cup c) \in R$

2. if $a^{\prime} \vdash_{A} a,(a, b) \in R$, and $b \vdash_{B} b^{\prime}$, then $\left(a^{\prime}, b^{\prime}\right) \in R$

Any approximable relation $R$ is uniquely determined by its trace $\operatorname{Tr}(R)=\{(a, \beta):(a,\{\beta\}) \in R\}$ in the sense that $R=\left\{\left(a,\left\{\beta_{1}, \ldots, \beta_{n}\right\}\right):\left(a, \beta_{1}\right), \ldots,\left(a, \beta_{n}\right) \in \operatorname{Tr}(R)\right\}$.

We will call Inf the category which has information systems as objects and traces of approximable relations as arrows; in this category the identity of an object $\mathcal{A}$ is its entailment relation $\vdash_{A}$. Note that any hom-set $\operatorname{Inf}(\mathcal{A}, \mathcal{B})$, ordered by inclusion, is a Scott domain.

The information system $(\emptyset,\{\emptyset\}, \emptyset)$ is the terminal object for this category and it is well-known that it is possible to define Cartesian products ( [20, Def. 6.1] (defined as disjoint unions $\uplus$ of information systems) and exponential objects ( [20, Def. 7.1]) in Inf in such a way that it constitutes a ccc. For later purposes, we define (up to iso in Inf) the exponent of $\mathcal{A}$ and $\mathcal{B}$ as $\mathcal{A} \Rightarrow \mathcal{B}=\left(\operatorname{Con}_{A} \times B\right.$, Con, $\left.\vdash\right)$, where

- $\left\{\left(a_{1}, \beta_{1}\right), \ldots,\left(a_{m}, \beta_{m}\right)\right\} \in \operatorname{Con}_{A \Rightarrow B}$ iff $\forall I \subseteq[1, m] . \cup_{i \in I} a_{i} \in \operatorname{Con}_{A} \Rightarrow\left\{\beta_{i}: i \in I\right\} \in \operatorname{Con}_{B}$

- $\left\{\left(a_{1}, \beta_{1}\right), \ldots,\left(a_{n}, \beta_{n}\right)\right\} \vdash(c, \delta)$ iff $\left\{\beta_{i}: c \vdash a_{i}\right\} \vdash \delta$.

The two categories SD of Scott domains and Inf of information systems are equivalent; to show this, define two functors $(-)^{-}: \mathbf{S D} \rightarrow \operatorname{Inf}$ and $(-)^{+}: \mathbf{I n f} \rightarrow \mathbf{S D}$ as follows:

- for $\mathcal{A} \in \operatorname{Obj}(\operatorname{Inf}), \mathcal{A}^{+}$is the Scott domain of all points of $\mathcal{A}$, ordered by inclusion;

- for $R \in \operatorname{Inf}(\mathcal{A}, \mathcal{B}), R^{+}(x)=\{\beta: \exists a \subseteq x$. $(a, \beta) \in$ $R\}\left(x \in \mathcal{A}^{+}\right)$. The continuous function $R^{+}$will be denoted by fun $(R)$.

- for $\mathcal{D} \in \operatorname{Obj}(\mathbf{S D}), \mathcal{D}^{-}=(\mathcal{K}(\mathcal{D})$, Con, $\vdash)$, where $a \in$ Con iff $a \subseteq_{\mathrm{f}} \mathcal{K}(\mathcal{D})$ is upper-bounded in $\mathcal{D}$; (ii) $a \vdash \alpha$ iff $\alpha \leq \sqcup a$.

- for $f \in \mathbf{S D}(\mathcal{D}, \mathcal{E}), f^{-}=\{(a, \beta): \beta \in f(\sqcup a)\}$. The trace $f^{-}$will be denoted by $\operatorname{gph}(f)$.

Proposition 9. The functors $(-)^{-}:$SD $\rightarrow$ Inf and $(-)^{+}:$Inf $\rightarrow$ SD are both full and faithful, and the two composite endofunctors $(-)^{-} \circ(-)^{+}$and $(-)^{+} \circ(-)^{-}$are naturally isomorphic to the identity functor of Inf and SD, respectively. Moreover the functors $(-)^{-}$and $(-)^{+}$preserve exponentials and products, i.e. $(\mathcal{A} \Rightarrow \mathcal{B})^{+} \cong\left[\mathcal{A}^{+} \rightarrow \mathcal{B}^{+}\right]$ and $(\mathcal{A} \uplus \mathcal{B})^{+} \cong \mathcal{A}^{+} \times \mathcal{B}^{+}$in the category $\mathrm{SD}$, and $[\mathcal{D} \rightarrow \mathcal{E}]^{-} \cong \mathcal{D}^{-} \Rightarrow \mathcal{E}^{-}$and $(\mathcal{D} \times \mathcal{E})^{-} \cong \mathcal{D}^{-} \uplus \mathcal{E}^{-}$in the category Inf. 
Proof: The family of arrows of Inf given by

$$
\left\langle\nu_{\mathcal{A}}: \mathcal{A} \rightarrow \mathcal{A}^{+-}: \mathcal{A} \in \mathbf{I n f}\right\rangle
$$

with components $\nu_{\mathcal{A}}=\{(a, \Downarrow b): b \subseteq \Downarrow a\}$ is a natural isomorphism. The family of arrows of SD given by

$$
\left\langle\mu_{\mathcal{D}}: \mathcal{D} \rightarrow \mathcal{D}^{-+}: \mathcal{D} \in \mathbf{S D}\right\rangle
$$

with components $\mu_{\mathcal{D}}(x)=\{\alpha \in \mathcal{K}(\mathcal{D}): \alpha \sqsubseteq \mathcal{D} x\}$ is another natural isomorphism.

$\operatorname{Inf}(\mathcal{A}, \mathcal{B})$ and $(\mathcal{A} \Rightarrow \mathcal{B})^{+}$are the same Scott domain. fun : $(\mathcal{A} \Rightarrow \mathcal{B})^{+} \cong\left[\mathcal{A}^{+} \rightarrow \mathcal{B}^{+}\right]$and gph $:[\mathcal{D} \rightarrow \mathcal{E}] \cong$ $\left(\mathcal{D}^{-} \Rightarrow \mathcal{E}^{-}\right)^{+}$are isomorphisms determined by the functors $(-)^{+}$and $(-)^{-}$restricted to the hom-sets. Clearly $[\mathcal{D} \rightarrow$ $\mathcal{E}]^{-+} \cong[\mathcal{D} \rightarrow \mathcal{E}] \cong\left(\mathcal{D}^{-} \Rightarrow \mathcal{E}^{-}\right)^{+}$in $\mathbf{S D}$; the conclusion easily follows.

From the correspondence given in Prop. 9 it follows that

(i) there exists a retraction (e.p.-, iso-) pair from $\mathcal{E}$ to $\mathcal{D}$ in SD iff there exists a retraction (e.p.-, iso-) pair from $\mathcal{E}^{-}$to $\mathcal{D}^{-}$in Inf.

(ii) $\mathcal{D}$ is a (semi-extensional, extensional) reflexive object in $\mathrm{SD}$ iff $\mathcal{D}^{-}$is a (semi-extensional, extensional) reflexive object in Inf.

\section{WEBBED MODELS FOR EXTENSIONAL REFLEXIVE SCOTT DOMAINS}

In this section we introduce a class of webbed models, called iweb-models, arising from information systems. In the main result of the section we show that iweb-models include, besides the filter models living in Cpo, all extensional reflexive Scott domains.

It is well-known that if $(F, G)$ is an e.p.-pair from $\mathcal{E}$ into $\mathcal{D}$ in $\mathbf{S D}$, then $F$ is uniquely determined by $G$, in the sense that $F(x)=\bigsqcup\{y \in E: G(y) \leq x\}$. The situation is even better in the category Inf, where an e.p.-pair, that is a suitable pair of (traces of) approximable relations, is determined by a unique function with suitable properties (see Lemma 13 below).

Definition 10. Let $\mathcal{A}, \mathcal{B}$ be information systems. A morphism $\phi$ is a function from $A$ into $B$ satisfying the following condition

$$
\text { (H1) } a \in \operatorname{Con}_{A} \Longleftrightarrow \phi(a) \in \operatorname{Con}_{B} .
$$

If $\phi: \mathcal{A} \rightarrow \mathcal{B}$ is a morphism, we define $\phi^{\rightarrow}: \mathcal{A}^{+} \rightarrow \mathcal{B}^{+}$ and $\phi^{\leftarrow}: \mathcal{B}^{+} \rightarrow \mathcal{A}^{+}$as follows:

- $\phi^{\rightarrow}(x)=\Downarrow_{B}\{\phi(\alpha): \alpha \in x\}$, for every point $x$ of $\mathcal{A}$;

- $\phi^{\leftarrow}(y)=\Downarrow_{A}\{\alpha: \phi(\alpha) \in y\}$, for every point $y$ of $\mathcal{B}$.

Lemma 11. $\phi^{\rightarrow}, \phi^{\leftarrow}$ are well defined and Scott continuous.

$\phi^{\rightarrow}$ and $\phi^{\leftarrow}$ are candidate to be a retraction, but we need more hypotheses.

Definition 12. We say that a morphism $\phi: \mathcal{A} \rightarrow \mathcal{B}$ is a:
1. backward morphism (b-morphism, for short) if it satisfies

$$
\text { (H2b) } \phi(a) \vdash_{B} \phi(\alpha) \Rightarrow a \vdash_{A} \alpha .
$$

2. forward morphism (f-morphism, for short) if it satisfies

$$
\text { (H2f) } a \vdash_{A} \alpha \Rightarrow \phi(a) \vdash_{B} \phi(\alpha) .
$$

3. bf-morphism if it is both a b-morphism and an fmorphism.

4. strong bf-morphism (sbf-morphism, for short) if it is a bf-morphism satisfying

$$
\text { (H3) } b \vdash_{B} \beta \Rightarrow b \cap \operatorname{ran}(\phi) \vdash_{B} \beta .
$$

The relativization of the various notions of morphism to the case in which $\phi$ is a partial map is obtained by requiring $a \in \operatorname{dom}(\phi)$ in (H1) and $a \cup\{\alpha\} \in \operatorname{dom}(\phi)$ in (H2f) and (H2b).

Lemma 13. (i) Let $\phi: \mathcal{A} \rightarrow \mathcal{B}$ be $a$ b- (bf-, sbf-) morphism. Then $\left(\phi^{\leftarrow}, \phi^{\rightarrow}\right)$ is a retraction (e.p.-, iso-) pair from $\mathcal{A}^{+}$to $\mathcal{B}^{+}$.

(ii) Let $(F, G)$ be an e.p.- (iso-) pair from $\mathcal{E}$ to $\mathcal{D}$. Then $G$ restricted to $\mathcal{K}(\mathcal{E})$ is a bf- (sbf-) morphism from $\mathcal{E}^{-}$ to $\mathcal{D}^{-}$.

Proof: (i) From (H2b) it follows $\phi^{\leftarrow} \circ \phi^{\rightarrow}=I d_{\mathcal{A}^{+}}$, while the relation $\phi^{\rightarrow} \circ \phi^{\leftarrow} \leq I d_{\mathcal{B}^{+}}$follows from (H2f).

(ii) By $G \circ F \leq i d_{\mathcal{D}}$ the map $G$ restricted to $\mathcal{K}(\mathcal{E})$ is an injection from $\mathcal{K}(\mathcal{E})$ into $\mathcal{K}(\mathcal{D})$; (H1) follows from $F \circ G=$ $i d_{\mathcal{E}}$ and the monotonicity of $F, G$. Finally, (H2b) and (H2f) are consequences of $F \circ G=i d_{\mathcal{E}}$ and the continuity of $G$, respectively.

Definition 14. A pair $\mathbf{A}=\left(\mathcal{A}, \phi_{A}\right)$ is called an iweb if $\mathcal{A}$ is an information system and $\phi_{A}:(\mathcal{A} \Rightarrow \mathcal{A}) \rightarrow \mathcal{A}$ is a b-morphism.

Partial iwebs are defined by partial b-morphisms. If $\mathbf{A}$ is a partial iweb, then we implicitly mean that $\mathbf{A}=\left(\mathcal{A}, \phi_{A}\right)$.

A is a finite iweb if it is a partial iweb and $A$ is a finite set.

Proposition 15. If $\mathbf{A}$ is an iweb, then $\mathbf{A}^{+}=\left(\mathcal{A}^{+}\right.$, fun $\circ$ $\left.\phi_{A}^{\leftarrow}, \phi_{A} \circ \mathrm{gph}\right)$ is a reflexive Scott domain.

Proof: By Lemma 13(i) and by Prop. 9.

Semi-extensional and extensional iwebs are respectively defined by bf-morphisms and sbf-morphisms. They generate respectively semi-extensional and extensional reflexive Scott domains.

Definition 16. Let $\mathbf{A}$ be an iweb. Then the reflexive Scott domain $\mathbf{A}^{+}$is called an iweb-model.

Let $\mathbf{A}$ be an iweb. The interpretation function $|-|^{\mathbf{A}}$ : $\Lambda \times E n v_{\mathcal{A}^{+}} \rightarrow \mathcal{A}^{+}$of $\lambda$-terms is defined inductively in the standard way: 
- $|y|_{\rho}^{\mathbf{A}}=\rho(y)$

- $|\lambda y \cdot M|_{\rho}^{\mathbf{A}}=\Downarrow\left\{\phi_{A}(a, \alpha): \alpha \in|M|_{\rho[y:=\Downarrow a]}^{\mathbf{A}}\right\}$

- $|M N|_{\rho}^{\mathbf{A}}=\left\{\beta: \exists a \subseteq|N|_{\rho}^{\mathbf{A}},(a, \beta) \in \phi_{A}^{\leftarrow}\left(|M|_{\rho}^{\mathbf{A}}\right)\right\}$.

Example 17. (Filter Models) Let $(A, \wedge)$ be a meetsemilattice. Then $\mathcal{A}=\left(A, \mathcal{P}_{\mathrm{f}}(A), \vdash\right)$, where $a \vdash \alpha$ iff $(\bigwedge a) \leq \alpha$, is an information system. If $\rightarrow$ is a binary operation on $A$, then define $\phi: \mathcal{P}_{\mathrm{f}}(A) \times A \rightarrow A$ by $\phi(a, \alpha)=(\bigwedge a) \rightarrow \alpha$. Map $\phi$ is a morphism, i.e., it satisfies (H1), because $\operatorname{Con}_{A}=\mathcal{P}_{\mathrm{f}}(A) .(\mathcal{A}, \phi)$ satisfies (H2b) (i.e., it is an iweb) iff the following property holds (see [10, Def. 2.12]): if $\bigwedge_{i=1}^{n}\left(\alpha_{i} \rightarrow \beta_{i}\right) \leq \gamma \rightarrow \delta$ then $\left(\bigwedge_{i \in\left\{i: \gamma \leq \alpha_{i}\right\}} \beta_{i}\right) \leq \delta$.

Example 18. (Preordered Coherent Models) A preordered set with coherence is a triple $(A, \leq, \approx)$, where $A$ is a nonempty set, $\leq$ is a preorder on $A$ and $\approx$ is a coherence (i.e., a reflexive, symmetric relation on $A$ ) compatible with the preorder (see [3, Def. 120]). A pc-information system is a triple $\mathcal{A}=(A$, Con,-$)$, where Con $=\mathcal{P}_{\mathrm{f}}^{\mathrm{coh}}(A)$ (the finite coherent subsets of $A$ ) and $a \vdash \alpha$ iff $\exists \beta \in a . \beta \geq \alpha$. A pcweb (see [3, Def. 153]) is determined by a pc-information system $\mathcal{A}$ and a b-morphism $\phi$ from $\mathcal{A} \Rightarrow \mathcal{A}$ to $\mathcal{A}$. This means that $\phi$ satisfies:

(H1) $\phi(a, \alpha) \approx \phi(b, \beta)$ iff $\left(a \cup b \in \mathcal{P}_{\mathrm{f}}^{\mathrm{coh}}(A) \Rightarrow \alpha \approx \beta\right)$

(H2b) if $\phi(a, \alpha) \leq \phi(b, \beta)$, then $\alpha \leq \beta$ and $(\forall \gamma \in b \exists \delta \in$ $a \cdot \gamma \leq \delta)$.

Clearly Krivine models of $\lambda$-calculus [3, Section 5.6.2] arise from $p c$-webs in which $\approx=A \times A$, while graph models [3, Section 5.5] from pc-webs in which $\approx=A \times A$ and $\leq$ is the equality.

Let $\mathcal{E}, \mathcal{D}$ be Scott domains. If $\alpha \in \mathcal{K}(\mathcal{E})$ and $\beta \in \mathcal{K}(\mathcal{D})$, we denote by $\alpha \rightsquigarrow \beta$ the step function from $\mathcal{E}$ into $\mathcal{D}$ defined by

$$
(\alpha \rightsquigarrow \beta)(x)=\text { if } x \geq \alpha \text { then } \beta \text { else } \perp_{\mathcal{D}} .
$$

Theorem 19. For every (semi-)extensional reflexive Scott domain $\mathbf{D}=(\mathcal{D}, F, G)$ there exists an iweb-model with the same equational and order theories of $\mathbf{D}$.

Proof: We prove the statement for the extensional case. First of all, we define a sbf-morphism $\nu$ of information systems from $\mathcal{D}^{-} \Rightarrow \mathcal{D}^{-}$onto $[\mathcal{D} \rightarrow \mathcal{D}]^{-}$as follows:

$$
\nu(a, \beta)=\sqcup a \rightsquigarrow \beta, \quad \text { for } a \in \operatorname{Con}_{\mathcal{D}^{-}} \text {and } \beta \in \mathcal{K}(\mathcal{D}) .
$$

It follows from Lemma 13(1) that the Scott domains $\left(\mathcal{D}^{-} \Rightarrow\right.$ $\left.\mathcal{D}^{-}\right)^{+}$and $[\mathcal{D} \rightarrow \mathcal{D}]^{-+}$are isomorphic via the map $\nu^{\rightarrow}$.

Define $\mathbf{A}=\left(\mathcal{D}^{-}, G \circ \nu\right)$. By Lemma 13(2) $G \circ \nu$ is a sbf-morphism, so that $\mathbf{A}$ is an extensional iweb and $\mathbf{A}^{+}$is an extensional reflexive Scott domain.

The $\lambda$-models $\mathbf{D}$ and $\mathbf{A}^{+}$are isomorphic because $\mu_{\mathcal{D}}$ is an isomorphism between the Scott domains $\mathcal{D}$ and $\mathcal{D}^{-+}$and $G=\mu_{\mathcal{D}}^{-1} \circ(G \circ \nu) \rightarrow \circ\left(\nu^{\rightarrow}\right)^{-1} \circ \mu_{[\mathcal{D} \rightarrow \mathcal{D}]}$, where all involved maps are isomorphisms. Recall the definition of $\mu$ from the proof of Prop. 9.

\section{COMPLETION OF PARTIAL IWEBS}

Starting from a partial iweb $\mathbf{A}$, it is possible to obtain by "completion" a total one which "extends" A. We apply this method to show that, if an inequality fails in an iweb-model, then it fails in an iweb-model arising from the canonical completion of a suitable finite iweb.

Let $\mathbf{A}$ be a partial iweb. We define an information system $\mathcal{A}^{*}$ extending $\mathcal{A}$ and a map $\phi^{*}: \operatorname{Con}_{A} \times A \rightarrow A^{*}$, which is a b-morphism from $\mathcal{A} \Rightarrow \mathcal{A}$ into $\mathcal{A}^{*}$ :

- $A^{*}=A \cup\left(\left(\operatorname{Con}_{A} \times A\right)-\operatorname{dom}\left(\phi_{A}\right)\right)$

- $\phi^{*}(\alpha)=$ if $\alpha \in \operatorname{dom}\left(\phi_{A}\right)$ then $\phi_{A}(\alpha)$ else $\alpha$

- $x \in \mathrm{Con}^{*}$ iff

- either $x \in \operatorname{Con}_{A}$

- or there are $X \in \operatorname{Con}_{A \Rightarrow A}$ and $b \subseteq_{\mathrm{f}} A \backslash \operatorname{ran}\left(\phi_{A}\right)$ such that $\phi^{*}(X) \cap A \in \operatorname{Con}_{A}, \phi^{*}(X) \cap A \vdash_{A} b$, and $x \subseteq \phi^{*}(X) \cup b$

- $a \vdash^{*} \alpha$ iff $a \in$ Con $^{*}$ and (either $a \cap A \vdash_{A} \alpha$ or $\alpha \in a$ )

Lemma 20. Let $\mathbf{A}$ be a partial iweb. Then $\mathcal{A}^{*}$ is an information system and $\phi^{*}$ is a b-morphism from $\mathcal{A} \Rightarrow \mathcal{A}$ to $\mathcal{A}^{*}$.

Proof: We proceed to verify all the axioms of information system. Clearly Con* contains all singletons, and $\emptyset \in \mathrm{Con}^{*}$ because $\emptyset \in \operatorname{Con}_{A}$. Moreover Con* is downward closed by definition. Axiom (IS1) is satisfied by definition of $\vdash^{*}$. For (IS2), assume $a \vdash^{*} b \vdash^{*} \alpha$. If $\alpha \in b$, then we have the conclusion; otherwise, we have that $b \cap A \vdash_{A} \alpha$. From $a \cap A \vdash_{A} b \cap A$, by applying property (IS2) for $\mathcal{A}$, we get $a \cap A \vdash_{A} \alpha$ and hence $a \vdash^{*} \alpha$. We now prove axiom (IS3). Assume $a \vdash^{*} \alpha$ : if $a \in \operatorname{Con}_{A}$ or $\alpha \in a$, then the result is obvious. Otherwise $a \cap A \vdash_{A} \alpha$ and there exist $X \in \operatorname{Con}_{A \Rightarrow A}$ and $b \subseteq_{\mathrm{f}} A \backslash \operatorname{ran}\left(\phi_{A}\right)$ such that $\phi^{*}(X) \cap A \in$ $\operatorname{Con}_{A}, \phi^{*}(X) \cap A \vdash_{A} b$, and $a \subseteq \phi^{*}(X) \cup b$. Then we get $\left(\phi^{*}(X) \cap A\right) \cup b \vdash_{A} \alpha$ and therefore $\phi^{*}(X) \cap A \vdash_{A}\{\alpha\} \cup b$, so that $a \cup\{\alpha\} \subseteq \phi^{*}(X) \cup b \cup\{\alpha\}$.

If $\alpha \notin \operatorname{ran}\left(\phi_{A}\right)$, then by definition of $\mathrm{Con}^{*}$ we have that $a \cup\{\alpha\} \in \mathrm{Con}^{*}$.

If $\alpha \in \operatorname{ran}\left(\phi_{A}\right)$, then $\alpha=\phi_{A}(c, \gamma)$ for some $c, \gamma$. Let $Y=X \cup\{(c, \gamma)\}$. Then we have $a \cup\{\alpha\} \subseteq \phi^{*}(Y) \cup b$. From $\phi^{*}(X) \cap A=\phi_{A}\left(X \cap \operatorname{dom}\left(\phi_{A}\right)\right)$ it follows that $\phi_{A}(X \cap$ $\left.\operatorname{dom}\left(\phi_{A}\right)\right) \vdash_{A} \phi_{A}(c, \gamma)$. Then by property (H2b) for $\phi_{A}$ we get $X \cap \operatorname{dom}\left(\phi_{A}\right) \vdash_{A \Rightarrow A}(c, \gamma)$ and thus $X \vdash_{A \Rightarrow A}(c, \gamma)$. We now conclude $a \cup\{\alpha\} \in \mathrm{Con}^{*}$ by noting that $Y \in \operatorname{Con}_{A \Rightarrow A}$, $\phi^{*}(Y) \cap A=\phi_{A}\left(Y \cap \operatorname{dom}\left(\phi_{A}\right)\right) \in \operatorname{Con}_{A}$ (by property (H1) for $\left.\phi_{A}\right)$, and finally $\phi^{*}(Y) \cap A \vdash_{A} b$.

This shows that $\mathcal{A}^{*}$ is an information system. We now prove properties (H1) and (H2b) for $\phi^{*}: \mathcal{A} \Rightarrow \mathcal{A} \rightarrow \mathcal{A}^{*}$, making it a b-morphism.

(H1) Assume $X \in \operatorname{Con}_{A \Rightarrow A}$. We have to show that $\phi^{*}(X) \in$ Con $^{*}$. Clearly $X \cap \operatorname{dom}\left(\phi_{A}\right) \in \operatorname{Con}_{A \Rightarrow A}$ and using $(\mathrm{H} 1)$ for $\phi_{A}$ we get that $\phi_{A}\left(X \cap \operatorname{dom}\left(\phi_{A}\right)\right)=$ $\phi^{*}(X) \cap A \in \operatorname{Con}_{A}$. The conclusion follows by the definition of Con* with $b=\emptyset$. 
Assume $\phi^{*}(X) \in \mathrm{Con}^{*}$. If $\phi^{*}(X) \in \operatorname{Con}_{A}$, then the conclusion follows immediately by (H1) for $\phi_{A}$. Otherwise there exist $Y \in \operatorname{Con}_{A \Rightarrow A}$ and $b \subseteq_{\mathrm{f}} A \backslash \operatorname{ran}\left(\phi_{A}\right)$ such that $\phi^{*}(Y) \cap A \in \operatorname{Con}_{A}, \phi^{*}(Y) \cap A \vdash_{A} b$ and $\phi^{*}(X) \subseteq \phi^{*}(Y) \cup$ $b$. From $b \subseteq A \backslash \operatorname{ran}\left(\phi_{A}\right)$ it follows that $\phi^{*}(X) \subseteq \phi^{*}(Y)$.

Since $\phi^{*}$ is the identity on all elements not in the domain of $\phi_{A}$, it follows that $X \backslash \operatorname{dom}\left(\phi_{A}\right) \subseteq Y \backslash \operatorname{dom}\left(\phi_{A}\right)$ and for every $(a, \alpha) \in X \cap \operatorname{dom}\left(\phi_{A}\right)$ there exists $(c, \gamma) \in Y \cap$ $\operatorname{dom}\left(\phi_{A}\right)$ such that $\phi_{A}(a, \alpha)=\phi_{A}(c, \gamma)$. By the property (H2b) of $\phi_{A}$ we derive $(a, \alpha)-\vdash_{A \Rightarrow A}(c, \gamma)$. It follows that $Y \vdash_{A \Rightarrow A}(a, \alpha)$ for all $(a, \alpha) \in X \cap \operatorname{dom}\left(\phi_{A}\right)$. This means that $Y \vdash_{A \Rightarrow A} X$, and hence $X \in \operatorname{Con}_{A \Rightarrow A}$.

(H2b) Assume that $\phi^{*}(X) \vdash^{*} \phi^{*}(\alpha)$. Since $\phi^{*}(X) \in$ Con*, by (H1) for $\phi^{*}$ we derive that $X \in \operatorname{Con}_{A \Rightarrow A}$. There are two cases to be dealt with.

If $\alpha \in \operatorname{dom}\left(\phi_{A}\right)$, then $\phi^{*}(X) \cap A \vdash_{A} \phi_{A}(\alpha)$ and we derive $\phi_{A}\left(X \cap \operatorname{dom}\left(\phi_{A}\right)\right) \vdash_{A} \phi_{A}(\alpha)$ so that by (H2b) for $\phi_{A}$ we have that $X \cap \operatorname{dom}\left(\phi_{A}\right) \vdash_{A \Rightarrow A} \alpha$ and hence $X \vdash_{A \Rightarrow A} \alpha$.

If $\alpha \notin \operatorname{dom}\left(\phi_{A}\right)$, then $\alpha=\phi^{*}(\alpha) \in \phi^{*}(X)$, so that $\alpha \in X$ and this concludes the proof.

Let $\mathbf{A}$ be a partial iweb, and assume $A$ does not contain any pairs. Define inductively $\left(\mathcal{A}_{n}, \phi_{n}\right)$ as follows: $\left(\mathcal{A}_{0}, \phi_{0}\right):=\left(\mathcal{A}, \phi_{A}\right) ;\left(\mathcal{A}_{n+1}, \phi_{n+1}\right):=\left(\mathcal{A}_{n}^{*}, \phi_{n}^{*}\right)$.

The canonical completion of $\mathbf{A}$ is the following structure $\mathcal{A}_{\omega}=\left(\mathcal{A}_{\omega}, \phi_{\omega}\right): \mathcal{A}_{\omega}:=\cup_{m \in \mathbb{N}} \mathcal{A}_{m}, \phi_{\omega}:=\bigcup_{m \in \mathbb{N}} \phi_{m}$. In the following we write $\operatorname{Con}_{\omega}$ and $\Downarrow_{\omega}$ for $\operatorname{Con}_{A_{\omega}}$ and $\Downarrow_{\mathcal{A}_{\omega}}$.

Proposition 21. If $\mathbf{A}$ is a partial iweb, then $\mathbf{A}_{\omega}$ is an iweb.

Proof: By Lemma $20 \phi_{n+1}: \mathcal{A}_{n} \Rightarrow \mathcal{A}_{n} \rightarrow \mathcal{A}_{n+1}$ is a b-morphism. Then $\phi_{\omega}: \mathcal{A}_{\omega} \Rightarrow \mathcal{A}_{\omega}=\left(\cup_{n \in \mathbb{N}} \mathcal{A}_{n} \Rightarrow \mathcal{A}_{n}\right) \rightarrow$ $\mathcal{A}_{\omega}=\cup_{n \in \mathbb{N}} \mathcal{A}_{n+1}$ is a b-morphism.

Definition 22. 1. The restriction of an information system $\mathcal{A}$ to $S \subseteq A$ is the information system $\mathcal{A} \uparrow_{S}=$ $\left(S, \mathrm{Con}_{S}, \vdash_{S}\right)$, where $\operatorname{Con}_{S}=\left\{a \in \operatorname{Con}_{A}: a \subseteq S\right\}$ and $\vdash_{S}=\vdash_{A} \cap \operatorname{Con}_{S} \times S$. $\mathcal{S}$ is said to be a subsystem of an information system $\mathcal{A}$ if $\mathcal{S}=\left.\mathcal{A}\right|_{S}$.

2. A partial iweb $\mathbf{S}$ is a subweb of an iweb $\mathbf{A}$ if $\mathcal{S}$ is a subsystem of $\mathcal{A}$, and $\phi_{S} \subseteq \phi_{A} \cap\left(\operatorname{Con}_{S} \times S\right) \times S$.

3. A morphism of iwebs from an iweb $\mathbf{B}=\left(\mathcal{B}, \phi_{B}\right)$ to an iweb $\mathbf{C}=\left(\mathcal{C}, \phi_{C}\right)$ is a morphism $g: \mathcal{B} \rightarrow \mathcal{C}$ of information systems satisfying

$$
\text { (H4) } g\left(\phi_{B}(a, \alpha)\right)=\phi_{C}(g(a), g(\alpha)) \text {. }
$$

Similarly for the other types of morphism.

Lemma 23. A morphism $g: \mathcal{B} \rightarrow \mathcal{C}$ of information systems induces a morphism $g \Rightarrow: \mathcal{B} \Rightarrow \mathcal{B} \rightarrow \mathcal{C} \Rightarrow \mathcal{C}$ by componentwise definition: $g \Rightarrow(b, \beta)=(g(b), g(\beta))$. Moreover we have that, if $g$ is a b-morphism (resp. f-morphism), then so is $g \Rightarrow$.

The following lemma holds with a very weak hypothesis.

Lemma 24. Let $\mathbf{B}$ and $\mathbf{C}$ be iwebs, and $h: B \rightarrow C$ be a function satisfying (H2f) and (H4). Then, for every $Z \in$
$\Lambda$, we have that $h\left(|Z|_{\rho}^{\mathbf{B}}\right) \subseteq|Z|_{h(\rho)}^{\mathbf{C}}$, where $h(\rho)(x)=\Downarrow_{C}$ $h(\rho(x))$.

Proof: The proof is by induction on the complexity of the $\lambda$-term $Z$.

$(Z \equiv P Q)$ If $\beta \in|P Q|_{\rho}^{\mathbf{B}}$, there exist $a \subseteq|Q|_{\rho}^{\mathbf{B}}$ and $X \in \operatorname{Con}_{B \Rightarrow B}$ such that $\phi_{B}(X) \subseteq|P|_{\rho}^{\mathbf{B}}$ and $X \vdash_{B \Rightarrow B}$ $(a, \beta)$. By (H4) and by the induction hypothesis we have that $h(a) \subseteq|Q|_{h(\rho)}^{\mathbf{C}}$ and $\phi_{C}(h \Rightarrow(X)) \subseteq|P|_{h(\rho)}^{\mathbf{C}}$.

If we prove that there is a subset $Y$ of $X$ such that $h^{\Rightarrow}(Y) \vdash_{\mathcal{C} \Rightarrow \mathcal{C}}(h(a), h(\alpha))$, then this last condition together with $h(a) \subseteq|Q|_{h \rho}^{\mathbf{C}}$ and $\phi_{C}(h \Rightarrow(Y)) \subseteq|P|_{h \rho}^{\mathbf{C}}$ imply that $h(\alpha) \in|P Q|_{h \rho}^{\mathrm{C}}$.

Since $X \vdash_{B \Rightarrow B}(a, \alpha)$ iff $\left\{\beta: \exists(b, \beta) \in X, a \vdash_{B} b\right\}$ $\vdash_{B} \alpha$, then by (H2f) we have that $\{h(\beta): \exists(b, \beta) \in$ $\left.X, a \vdash_{B} b\right\} \vdash_{C} h(\alpha)$. But by applying again (H2f) we get $\left\{h(\beta): \exists(b, \beta) \in X, a \vdash_{B} b\right\} \subseteq\{h(\beta): \exists(h(b), h(\beta)) \in$ $\left.h \Rightarrow(X), h(a) \vdash_{C} h(b)\right\}$. We get the conclusion by putting $Y=\left\{(b, \beta) \in X: a \vdash_{B} b\right\}$.

$(Z \equiv \lambda y . P)$ Clearly $\alpha \in|P|_{\rho\left[y:=\Downarrow_{B} a\right]}^{\mathrm{B}}$ implies $h(\alpha) \in$ $h\left(|P|_{\rho\left[y:=\Downarrow_{B} a\right]}^{\mathrm{B}}\right)$. Moreover, by $(\mathrm{H} 2 \mathrm{f}) h\left(\Downarrow_{B} a\right) \subseteq \Downarrow_{C} h(a)$, so that it holds:

$(*) \quad h\left(\rho\left[y:=\Downarrow_{B} a\right]\right) \subseteq h(\rho)\left[y:=\Downarrow_{C} h(a)\right]$

Hence we can proceed as follows:

$h\left(|\lambda y \cdot P|_{\rho}^{\mathbf{B}}\right)=h\left(\Downarrow_{B}\left\{\phi_{B}(a, \alpha): \alpha \in|P|_{\rho\left[y:=\Downarrow_{B} a\right]}^{\mathbf{B}}\right\}\right) \subseteq$ $\Downarrow_{C}\left\{\phi_{C}(h(a), h(\alpha)): h(\alpha) \in h\left(|P|_{\rho\left[y:=\Downarrow_{B} a\right]}^{\mathbf{B}}\right)\right\}$ by $(\mathrm{H} 2 \mathrm{f})-(\mathrm{H} 4)$

$\subseteq \Downarrow_{C}\left\{\phi_{C}(h(a), h(\alpha)): h(\alpha) \in|P|_{h\left(\rho\left[y:=\Downarrow_{C} h(a)\right]\right)}^{\mathbf{C}}\right\}$ by induction hypothesis and $(*)$

$\subseteq|\lambda y \cdot P|_{h(\rho)}^{\mathbf{C}}$

The following is the main theorem of the section.

Theorem 25. Let $\mathbf{A}$ be an iweb and $M \sqsubseteq N$ be an inequality which fails in the iweb-model $\mathbf{A}^{+}$. Then there is a finite subweb $\mathbf{S}$ of $\mathbf{A}$ such that $M \sqsubseteq N$ fails in the iweb-model $\mathbf{S}_{\omega}^{+}$arising from the canonical completion $\mathbf{S}_{\omega}$ of $\mathbf{S}$.

Proof: For an $A$-environment $\rho$, and $S \subseteq A$, we let $\rho \cap S$ be the $S$-environment defined by $(\rho \cap S)(x)=\rho(x) \cap S$.

Let $\alpha \in|M|_{\rho}^{\mathbf{A}} \backslash|N|_{\rho}^{\mathbf{A}}$ for some $A$-environment $\rho$.

Claim 26. There exists a finite subweb $\mathbf{S}$ of $\mathbf{A}$ such that $\alpha \in|M|_{\rho \cap S}^{\mathbf{S}}$.

Proof: The proof is by induction on the structure of $M$ by showing that $\alpha \in|M|_{\rho}^{\mathbf{A}}$ depends only on a finite number of elements of $A$. For example, if $M \equiv P Q$ and $\alpha \in|P Q|_{\rho}^{\mathbf{A}}$, then there exist $a \subseteq|Q|_{\rho}^{\mathbf{A}}$ and $c_{i}, \gamma_{i}(i \leq n)$ such that $\phi_{A}\left(c_{i}, \gamma_{i}\right) \in|P|_{\rho}^{\mathbf{A}}$ with $\left\{\left(c_{1}, \gamma_{1}\right), \ldots,\left(c_{n}, \gamma_{n}\right)\right\} \vdash_{A \Rightarrow A}$ $(a, \alpha)$. By induction hypothesis for each pair $\left(c_{i}, \gamma_{i}\right)$ there exists a finite subweb $\mathbf{S}_{i}$ of $\mathcal{A}$ such that $\phi_{A}\left(c_{i}, \gamma_{i}\right) \in|P|_{\rho \cap S_{i}}^{\mathbf{S}_{i}}$ and for all $\delta \in a$, there exists a finite subweb $\mathbf{S}_{\delta}$ of $\mathcal{A}$ such that $\delta \in|Q|_{\rho \cap S_{\delta}}^{\mathbf{S}_{\delta}}$. Then we set $S=\left(\bigcup_{i=1}^{n} S_{i}\right) \cup$ $\left(\bigcup_{\delta \in a} S_{\delta}\right) \cup\left(\bigcup_{i=1}^{n} c_{i}\right) \cup\left\{\alpha, \gamma_{1}, \ldots, \gamma_{n}\right\}, \mathcal{S}=\mathcal{A} \uparrow_{S}$; finally 
we put $\phi_{S}$ equal to $\phi_{A}$ restricted to set $\left(\bigcup_{i=1}^{n} \operatorname{dom}\left(\phi_{S_{i}}\right)\right) \cup$ $\left(\bigcup_{\delta \in a} \operatorname{dom}\left(\phi_{S_{\delta}}\right)\right) \cup\left\{\left(c_{1}, \gamma_{1}\right), \ldots,\left(c_{n}, \gamma_{n}\right)\right\}$.

Claim 27. There exists an f-morphism $g_{\omega}$ from the canonical completion $\mathbf{S}_{\omega}$ of $\mathbf{S}$ into $\mathbf{A}$.

Proof: As usual $\mathcal{S}_{0}=\mathcal{S}$ and $\mathcal{S}_{n+1}$ is the $(n+1)$-st information system produced by the canonical completion procedure of $\mathcal{S}$. We define $g_{\omega}=\cup_{n \in \mathbb{N}} g_{n}$, where $g_{n}: S_{n} \rightarrow$ $A$ is inductively defined as follows:

- $g_{0}=\mathrm{id}_{S}$;

- $g_{n+1}(\alpha)= \begin{cases}g_{n}(\alpha) & \text { if } \alpha \in S_{n} \\ \phi_{A}\left(g_{n}^{\Rightarrow}(b, \beta)\right) & \text { if } \alpha=(b, \beta) \in S_{n+1} \backslash S_{n}\end{cases}$

(H2f): Straightforward to verify, since $\vdash_{n}$ coincides with $\in$ for elements of $S_{\omega} \backslash S$.

(H4): If $(a, \alpha) \notin \operatorname{dom}\left(\phi_{S}\right), g_{\omega}\left(\phi_{\omega}(a, \alpha)\right)=g_{\omega}(a, \alpha)$ $=\phi_{A}\left(g_{\omega}(a), g_{\omega}(\alpha)\right)$. If $(a, \alpha) \in \operatorname{dom}\left(\phi_{S}\right)$ then $g_{\omega}$ is the identity on $S$ and $\phi_{\omega}=\phi_{S}=\phi_{A}$ restricted to $\operatorname{dom}\left(\phi_{S}\right)$.

(H1): We show by induction that $g_{n}: \mathcal{S}_{n} \rightarrow \mathcal{A}$ is a morphism of information systems. The condition trivially holds for $g_{0}$. Assume that $g_{n}: \mathcal{S}_{n} \rightarrow \mathcal{A}$ is a morphism. Then by Lemma $23 g_{n}^{\Rightarrow}: \mathcal{S}_{n} \Rightarrow \mathcal{S}_{n} \rightarrow \mathcal{A} \Rightarrow \mathcal{A}$ is a morphism too. Since $g_{n+1} \circ \phi_{n+1}=\phi_{A} \circ g_{n}^{\Rightarrow}$ we have: $\phi_{n+1}(a) \in \operatorname{Con}_{S_{n+1}}$ iff $a \in \operatorname{Con}_{S_{n} \Rightarrow S_{n}}$ iff $g_{n}^{\Rightarrow}(a) \in \operatorname{Con}_{A \Rightarrow A}$ iff $\phi_{A}\left(g_{n}^{\Rightarrow}(a)\right)=$ $g_{n+1}\left(\phi_{n+1}(a)\right) \in \operatorname{Con}_{A}$.

Claim 28. $\alpha \in|M|_{\rho \cap S}^{\mathbf{S}_{\omega}} \backslash|N|_{\rho \cap S}^{\mathbf{S}_{\omega}}$.

Proof: Recall that $\alpha \in S$ and that $\alpha \in|M|_{\rho \cap S}^{\mathbf{S}_{\omega}}$ by Claim 26. Assume, by the way of contradiction, that $\alpha \in|N|_{\rho \cap S}^{\mathbf{S}_{\omega}}$. Then from Lemma 24 it follows that $g_{\omega}(\alpha) \in$ $|N|_{g_{\omega}(\rho \cap S)}^{\mathbf{A}}$, that is, $\alpha \in|N|_{\rho \cap S}^{\mathbf{A}} \subseteq|N|_{\rho}^{\mathbf{A}}$, because $g_{\omega}$ is the identity restricted to $S$. This contradicts the original hypothesis.

This concludes the proof of Thm. 25 .

\section{EFFECTIVE INFORMATION SYSTEMS}

Definition 29. An effective information system is pair $(\mathcal{A}, \sigma)$, where $\mathcal{A}$ is an information system and $(A, \sigma)$ is a numeration such that the predicates Con and $\vdash$ are both $\sigma$-decidable.

Lemma 30. Let $(\mathcal{A}, \sigma)$ and $(\mathcal{B}, \tau)$ be effective information systems. Then there are a numbering $\sigma^{+}$of the compact points of $\mathcal{A}$ and a numbering $\sigma \Rightarrow \tau$ of $\operatorname{Con}_{A} \times B$ such that

(i) $\left(\mathcal{A}^{+}, \sigma^{+}\right)$is an effective Scott domain.

(ii) $(\mathcal{A} \Rightarrow \mathcal{B}, \sigma \Rightarrow \tau)$ is an effective information system.

(iii) $\left(\left[\mathcal{A}^{+} \rightarrow \mathcal{B}^{+}\right]\right.$, fun $\left.\circ(\sigma \Rightarrow \tau)^{+}\right)$is an effective Scott domain.

Proof: Let $\widehat{\sigma}: \mathbb{N} \rightarrow \mathcal{P}_{\mathrm{f}}(A)$ be the standard numbering obtained from $\sigma$ (see Section II-B).

(i) We define a numbering $\sigma^{+}$of the compact elements of $\mathcal{A}^{+}$by

$$
\sigma_{n}^{+}=\text {if } \widehat{\sigma}_{n} \in \operatorname{Con}_{A} \text { then } \Downarrow \widehat{\sigma}_{n} \text { else } \Downarrow \emptyset .
$$

It is now clear that for two compact points $\sigma_{n}^{+}, \sigma_{k}^{+}$we have: $\sigma_{n}^{+}, \sigma_{k}^{+}$compatible $\Longleftrightarrow \widehat{\sigma}_{n} \cup \widehat{\sigma}_{k} \in \operatorname{Con}_{A}$; hence the relation of compatibility is $\sigma^{+}$-decidable, since $\operatorname{Con}_{A}$ is $\sigma$ decidable. For three compact points $\sigma_{n}^{+}, \sigma_{m}^{+}$and $\sigma_{k}^{+}$we have: $\sigma_{n}^{+} \sqcup \sigma_{m}^{+}=\sigma_{k}^{+}$iff $\widehat{\sigma}_{n} \cup \widehat{\sigma}_{m} \in \operatorname{Con}_{A}$ and $\widehat{\sigma}_{k} \dashv\left(\widehat{\sigma}_{n} \cup\right.$ $\left.\widehat{\sigma}_{m}\right)$ and thus the relation of supremum is $\sigma^{+}$-decidable, since the right-hand side is $\sigma$-decidable.

(ii) Recall the bijective coding $\left\langle_{-},{ }_{-}\right\rangle$of Section II-B and define: $\xi_{\langle n, k\rangle}=$ if $\widehat{\sigma}_{n} \in \operatorname{Con}_{A}$ then $\left(\widehat{\sigma}_{n}, \tau_{k}\right)$ else $\left(\emptyset, \tau_{k}\right)$.

(iii) By (i)-(ii) we have that $\left((\mathcal{A} \Rightarrow \mathcal{B})^{+},(\sigma \Rightarrow \tau)^{+}\right)$is an effective Scott domain. Then the conclusion follows from Prop. 9, where it is defined an isomorphism fun : $(\mathcal{A} \Rightarrow$ $\mathcal{B})^{+} \cong\left[\mathcal{A}^{+} \rightarrow \mathcal{B}^{+}\right]$.

From now on whenever $(\mathcal{A}, \sigma)$ is an effective information system, then we will always consider the numbering $\sigma^{+}$ described in Lemma 30 as a standard one for the domain $\mathcal{A}^{+}$.

For every point $x \in \mathcal{A}^{+}$we have that $\left\{n: \sigma_{n}^{+} \subseteq x\right\}$ is r.e.

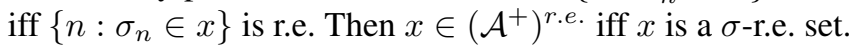
For any $\sigma$-r.e. consistent subset $x$ of $A, \Downarrow_{A} x \in\left(\mathcal{A}^{+}\right)^{r . e .}$.

Lemma 31. If $(\mathcal{D}, \sigma)$ is an effective Scott domain, then $\left(\mathcal{D}^{-}, \sigma\right)$ is an effective information system.

The following corollary provides an easy proof of a wellknown result.

Corollary 32. If $(\mathcal{D}, \sigma)$ and $(\mathcal{E}, \tau)$ are effective Scott domains, then $[\mathcal{D} \rightarrow \mathcal{E}]$ is an effective Scott domain too.

Proof: By Lemma 30(iii) and Lemma 31 we have that $\left(\left[\mathcal{D}^{-+} \rightarrow \mathcal{E}^{-+}\right]\right.$, fun $\left.\circ(\sigma \Rightarrow \tau)^{+}\right)$is an effective Scott domain. Then the conclusion follows from $\mathcal{D} \cong \mathcal{D}^{-+}$.

Lemma 33. Let $(\mathcal{A}, \sigma)$ and $(\mathcal{B}, \tau)$ be effective information systems. If $\phi: \mathcal{A} \rightarrow \mathcal{B}$ is a $(\sigma, \tau)$-computable morphism,

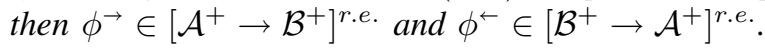

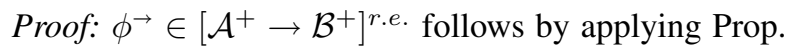
4(iii) to $\phi^{\rightarrow} \uparrow\left(\mathcal{A}^{+}\right)^{\text {r.e. }}$. Similarly for $\phi^{\leftarrow}$.

\section{EFFECTIVE IWEB-MODELS}

Definition 34. A (partial) iweb $\mathbf{A}$ is effective if there exists a numbering $\sigma$ of $A$ such that $(\mathcal{A}, \sigma)$ is an effective information system, $\phi_{A}: \mathcal{A} \Rightarrow \mathcal{A} \rightarrow \mathcal{A}$ is a strongly $(\sigma \Rightarrow \sigma, \sigma)$ computable function and $\operatorname{ran}\left(\phi_{A}\right)$ is $\sigma$-decidable.

Theorem 35. If a partial iweb is effective, then its canonical completion is effective too.

Proof: Let $(\mathbf{A}, \sigma)$, where $A \neq \emptyset$, be an effective partial iweb and consider the sequence $\left(\mathcal{A}_{n}, \phi_{n}\right)_{n \leq \omega}$ defined in the canonical completion procedure, where $\left(\mathcal{A}_{0}, \phi_{0}\right):=$ $\left(\mathcal{A}, \phi_{A}\right)$. We recall that $\mathcal{A}_{n}$ is a subsystem of $\mathcal{A}_{n+1}$ and that $\phi_{n+1}: \mathcal{A}_{n} \Rightarrow \mathcal{A}_{n} \rightarrow \mathcal{A}_{n+1}$ is a b-morphism. Now set $\sigma^{(0)}:=\sigma$ and assume that a sequence $\sigma^{(0)}, \ldots, \sigma^{(n)}$ of numberings, with $n \geq 0$, has already been constructed in such a way that $\left(\mathcal{A}_{n}, \sigma^{(n)}\right)$ is an effective information 
system, $\phi_{n}$ is strongly $\left(\sigma^{(n-1)} \Rightarrow \sigma^{(n-1)}, \sigma^{(n)}\right)$-computable and $\operatorname{ran}\left(\phi_{n}\right)$ is $\sigma^{(n)}$-decidable. Then it is routine to devise a numbering for $\left(\mathrm{Con}_{n} \times A_{n}\right) \backslash \operatorname{dom}\left(\phi_{n}\right)$ and from it to produce a numeration $\left(A_{n+1}, \sigma^{(n+1)}\right)$ in such a way that it is possible to decide whether a given element $\sigma_{m}^{(n+1)}$ belongs to $A_{n+1} \backslash A_{n}$ or to $A_{n}$, e.g., assigning to even numbers the elements of $A_{n}$ and to odd numbers the "new" pairs from $\left(\operatorname{Con}_{n} \times A_{n}\right) \backslash \operatorname{dom}\left(\phi_{n}\right)$.

Now $\phi_{n+1}$ extends $\phi_{n}$ mapping pairs to themselves and $\vdash_{n+1}$ extends $\vdash_{n}$ with membership, so that it is not difficult to show that $\phi_{n+1}$ is strongly $\left(\sigma^{(n)} \Rightarrow \sigma^{(n)}, \sigma^{(n+1)}\right)$ computable, $\operatorname{ran}\left(\phi_{n+1}\right)$ is $\sigma^{(n+1)}$-decidable and $\vdash_{n+1}$ is $\sigma^{(n+1)}$-decidable. Now a quick look at the definition of $\mathrm{Con}_{n+1}$ allows to conclude that this predicate is indeed $\sigma^{(n+1)}$-decidable.

Let $p_{n}$ be the $(n+1)$-st prime natural number. We now construct a numeration $\left(A_{\omega}, \zeta\right)$ as follows:

$$
\zeta_{m}=\text { if } m=\left(p_{n}\right)^{i} \text { then } \sigma_{i}^{(n)} \text { else } \sigma_{m}^{(m)}
$$

By using the corresponding properties of $\phi_{n}, \vdash_{n}$ and $\operatorname{Con}_{n}$, it is not difficult then to see that $\operatorname{dom}\left(\phi_{\omega}\right), \vdash_{\omega}$ and $\operatorname{Con}_{\omega}$ are all $\zeta$-decidable and $\phi_{\omega}$ is strongly $\zeta$-computable. Now since $A_{\omega} \backslash \operatorname{ran}\left(\phi_{\omega}\right)=A \backslash \operatorname{ran}\left(\phi_{A}\right)$, it is clear that $\operatorname{ran}\left(\phi_{\omega}\right)$ is $\zeta$-decidable. It follows that $\mathbf{A}_{\omega}$ is an effective iweb.

Lemma 36. Let $\mathbf{A}_{\omega}$ be the canonical completion of some finite iweb A. Then for any decidable consistent subset $x$ of $A_{\omega}, \Downarrow_{\omega} x \in\left(\mathcal{A}_{\omega}^{+}\right)^{\text {dec }}$.

Proof: Let $x \subseteq A_{\omega}$ be a consistent decidable set. By definition of $\mathcal{A}_{\omega}$ we have that $\Downarrow_{\omega} x=x \cup \Downarrow_{A}(x \cap A)$ and hence $\alpha \in \Downarrow_{\omega} x$ iff $\alpha \in x \vee\left(\alpha \in A \wedge \exists a \in \operatorname{Con}_{A} \cap x . a \vdash_{A}\right.$ $\alpha)$ The above predicate is clearly recursive, since $\operatorname{Con}_{A}$ is a finite set and the predicate $\alpha \in A$ is $\zeta$-decidable (by (1) above $\zeta_{m} \in A$ iff $m$ is a power of 2).

Theorem 37. If $(\mathbf{A}, \sigma)$ is an effective iweb, then $\left(\mathbf{A}^{+}, \sigma^{+}\right)$ is a weakly effective reflexive Scott domain.

Proof: By Lemma $30\left(\mathcal{A}^{+}, \sigma^{+}\right)$is an effective Scott domain, while by Lemma $33 \phi_{\vec{A}} \in\left[(\mathcal{A} \Rightarrow \mathcal{A})^{+} \rightarrow \mathcal{A}^{+}\right]^{\text {r.e. }}$. Since gph : $(\mathcal{A} \Rightarrow \mathcal{A})^{+} \cong\left[\mathcal{A}^{+} \rightarrow \mathcal{A}^{+}\right]$is an isomorphism, then we get that $G=\phi_{A} \circ$ gph $\in\left[\left[\mathcal{A}^{+} \rightarrow \mathcal{A}^{+}\right] \rightarrow \mathcal{A}^{+}\right]^{r . e .}$. Similarly for $F=$ fun $\circ \phi_{A}^{\leftarrow}$.

Lemma 38. Let $\mathbf{A}_{\omega}$ be the canonical completion of a finite iweb A. Then $|\lambda x . x|^{\mathbf{A}_{\omega}^{+}} \in\left(\mathcal{A}_{\omega}^{+}\right)^{d e c}$.

Proof: The finite iweb $\mathbf{A}$ is effective and hence, by Thm. 35, so is its canonical completion $\mathbf{A}_{\omega}$. Then by Thm. $37, \mathbf{A}_{\omega}^{+}$is a weakly effective reflexive Scott domain. Then we have:

$$
\begin{aligned}
|\lambda x . x|^{\mathbf{A}_{\omega}^{+}} & =\Downarrow_{\omega}\left\{\phi_{A_{\omega}}(a, \alpha): a \vdash_{A_{\omega}} \alpha\right\} \\
& =\Downarrow_{A}\left\{\phi_{A}(a, \alpha): a \vdash_{A} \alpha,(a, \alpha) \in \operatorname{dom}\left(\phi_{A}\right)\right\} \\
& \cup\left\{(b, \beta): \beta \in b,(b, \beta) \notin \operatorname{dom}\left(\phi_{A}\right)\right\} \\
& \cup\left\{(c, \gamma): c \cap A \vdash_{A} \gamma,(c, \gamma) \notin \operatorname{dom}\left(\phi_{A}\right)\right\}
\end{aligned}
$$

The conclusion follows from the last equality recalling that $A$ is finite.

Theorem 39. Let $\mathbf{A}_{\omega}$ be the canonical completion of a finite iweb A. Then $\operatorname{Ord}\left(\mathbf{A}_{\omega}^{+}\right)$is not r.e. and $\operatorname{Eq}\left(\mathbf{A}_{\omega}^{+}\right) \neq \lambda \beta, \lambda \beta \eta$.

Proof: By Thm. $37 \mathbf{A}_{\omega}^{+}$is a weakly effective $\lambda$-model and by Lemma $38|\lambda x . x|^{\mathbf{A}_{\omega}^{+}} \in\left(\mathcal{A}_{\omega}^{+}\right)^{d e c}$. Hence we conclude by Thm. 7(ii).

\section{THE MAIN THEOREMS}

The following lemma is important to obtain our main theorems.

Lemma 40. (i) (Scott [1, Thm. 6.6.4]) Let $X \subseteq \Lambda^{\circ}$ be $\beta$-closed and non-trivial (i.e., $\neq \emptyset, \Lambda^{\circ}$ ). Then $X$ is not decidable.

(ii) (Visser [1, Prop. 17.1.14]) No $\beta$-closed r.e. set of $\lambda$ terms different from $\Lambda$ contains a non-empty $\beta$-closed co-r.e. set of $\lambda$-terms.

Recall from [1] that the class of $\lambda$-models is closed under the construction of Cartesian product. Then the product $\mathbf{D}=$ $\prod_{k \in \mathbb{N}} \mathbf{D}_{k}$ of a countable family of reflexive Scott domains is a $\lambda$-model (although it is not a reflexive object in SD) such that $\operatorname{Eq}(\mathbf{D})=\bigcap_{k \in \mathbb{N}} \operatorname{Eq}\left(\mathbf{D}_{k}\right)$ and $\operatorname{Ord}(\mathbf{D})=\bigcap_{k \in \mathbb{N}} \operatorname{Ord}\left(\mathbf{D}_{k}\right)$.

Let $\mathbf{D}$ be a partially ordered $\lambda$-model and $M \in \Lambda^{\circ}$. Then we define: $M_{\downarrow}^{\mathbf{D}}=\left\{N \in \Lambda^{o}:|N|^{\mathbf{D}} \sqsubseteq \mathcal{D}|M|^{\mathbf{D}}\right\}$.

Lemma 41. Let $\mathbf{A}_{k}=\left(\mathcal{A}_{k}, \phi_{k}\right)(k \in \mathbb{N})$ be the family of all finite iwebs with $A_{k} \subseteq_{\mathrm{f}} \mathbb{N}$, let $\mathbf{A}_{k, \omega}(k \in \mathbb{N})$ be the family of their canonical completions and let $\mathbf{P}=\prod_{k \in \mathbb{N}} \mathbf{A}_{k, \omega}^{+}$be the Cartesian product of the reflexive Scott domains $\mathbf{A}_{k, \omega}^{+}$. Then

(i) $\mathcal{P}=\prod_{k \in \mathbb{N}} \mathcal{A}_{k, \omega}^{+}$is an effective Scott domain;

(ii) $|\lambda x . x|^{\mathbf{P}} \in \mathbf{P}^{\text {dec }}$ and $(\lambda x . x)_{\downarrow}^{\mathbf{P}}$ is a co-r.e. set;

(iii) $\operatorname{Ord}(\mathbf{P})$ is not r.e.

Proof: (i) It is not difficult to define an effective bijective numbering of all the finite iwebs whose carrier set is a finite subset of $\mathbb{N}$. Let $\mathbf{B}=\uplus_{k \in \mathbb{N}} \mathbf{A}_{k, \omega}$. Recalling Prop. 9, the product $\mathcal{P}$ is isomorphic to $\mathcal{B}^{+}$. Now consider an effective bijective function from $\mathbb{N}$ onto the set $B=\uplus_{k \in \mathbb{N}} A_{k, \omega}$ making $\mathcal{B}$ an effective information system. By Lemma 30 $\mathcal{B}^{+}$is an effective Scott domain. Then $\mathcal{P}$ is an effective Scott domain too.

(ii) Every element $x$ of the effective domain $\mathcal{P}$ is a sequence $\left(x_{0}, x_{1}, \ldots, x_{k}, \ldots\right)$ of sets which, without loss of generality, we can represent by the set $\uplus_{k \in \mathbb{N}} x_{k}$, where $x_{k}$ is a point in the Scott domain $\mathcal{A}_{k, \omega}^{+}$. Hence, we write $|M|^{\mathbf{P}}=\uplus_{k \in \mathbb{N}}|M|^{\mathbf{A}_{k, \omega}}$, where $|M|^{\mathbf{A}_{k, \omega}}$ is the interpretation of $M$ in the reflexive Scott domain $\mathbf{A}_{k, \omega}^{+}$.

Now we show that $|\lambda x . x|^{\mathbf{P}}=\uplus_{k \in \mathbb{N}}|\lambda x . x|^{\mathbf{A}_{k, \omega}} \in \mathbf{P}^{\text {dec }}$. Let $\alpha \in \uplus_{k \in \mathbb{N}} A_{k, \omega}$. First find the unique $k \in \mathbb{N}$ such that $\alpha \equiv\left(k, \alpha^{\prime}\right)$ and $\alpha^{\prime} \in A_{k, \omega}$. By Lemma 38 we have that

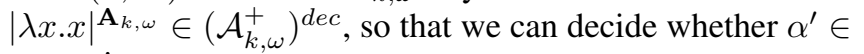
$|\lambda x . x|^{\mathbf{A}_{k, \omega}}$ or not. 
Finally, the set $(\lambda x \cdot x)_{\downarrow}^{\mathbf{P}}$ is co-r.e., because $|M|^{\mathbf{P}} \nsubseteq \mathbf{P}$ $|\lambda x . x|^{\mathbf{P}}$ iff $\exists k|M|^{\mathbf{A}_{k, \omega}} \nsubseteq|\lambda x . x|^{\mathbf{A}_{k, \omega}}$ and this last relation is r.e. again by Lemma 38 .

(iii) If $\operatorname{Ord}(\mathbf{P})$ is r.e., by (ii) the set $(\lambda x . x)_{\downarrow}^{\mathbf{P}}$ is $\beta$-closed and decidable. From Lemma 40(i) it follows that $(\lambda x . x)^{\mathbf{P}}=$ $\Lambda^{o}$. Then $|\lambda x y . y|^{\mathbf{P}} \sqsubseteq_{\mathbf{P}}|\lambda y . y|^{\mathbf{P}}$, that implies $|\lambda y . y|^{\mathbf{P}} \sqsubseteq_{\mathbf{P}}$ $|M|^{\mathbf{P}}$ for all $M \in \Lambda^{o}$. Hence, the theory of $\mathbf{P}$ is inconsistent. Contradiction.

Theorem 42. The order theory of an iweb-model is never r.e. and its equational theory is never equal to $\lambda \beta, \lambda \beta \eta$. In particular, this result applies to preordered coherent models and to those filter models which live in Cpo.

Proof: By Lemma 41 the order theory $\operatorname{Ord}(\mathbf{P})$ of the product $\mathbf{P}$ (as defined in Lemma 41) is not r.e. Now let $\mathbf{A}$ be an arbitrary iweb: it is clear that $\operatorname{Ord}(\mathbf{P}) \subseteq \operatorname{Ord}\left(\mathbf{A}^{+}\right)$, since by Thm. 25 any inequation between $\lambda$-terms which fails in $\mathbf{A}^{+}$, fails in a reflexive Scott domain arising from the canonical completion of a finite iweb, and then in $\mathbf{P}$ too. If $\operatorname{Ord}\left(\mathbf{A}^{+}\right)$is r.e., then $(\lambda x . x)_{\downarrow}^{\mathbf{A}}$ is a $\beta$-closed r.e. set of $\lambda$-terms containing $(\lambda x . x)_{\downarrow}^{\mathbf{P}}$; by Lemma 41(ii) the latter set is a $\beta$-closed co-r.e set: this leads to a contradiction of Lemma 40(ii). In conclusion, $\operatorname{Ord}\left(\mathbf{A}^{+}\right)$is not r.e. Because of this, if $\operatorname{Eq}\left(\mathbf{A}^{+}\right)$is r.e. then $\operatorname{Ord}\left(\mathbf{A}^{+}\right)$strictly contains $\mathrm{Eq}\left(\mathbf{A}^{+}\right)$; hence $\operatorname{Ord}\left(\mathbf{A}^{+}\right)$is nontrivial on the interpretation of $\lambda$-terms. The conclusion follows from Selinger's result stating that in any partially ordered $\lambda$-model, whose theory is $\lambda \beta(\lambda \beta \eta)$, the interpretations of distinct closed terms are incomparable [21, Cor. 4].

Corollary 43. The order theory of an extensional reflexive Scott domain is never r.e. and its equational theory is never equal to $\lambda \beta \eta$.

Still, the intersection of all $\lambda$-theories arising as equational theories of extensional reflexive Scott domains might be $\lambda \beta \eta$. This case is not possible as shown in the next theorem.

Theorem 44. Extensional reflexive Scott domains are not complete for the $\lambda \beta \eta$-calculus, i.e., there are equations not in $\lambda \beta \eta$ which hold in all extensional reflexive Scott domains.

Proof: Let $\mathbf{P}$ be the $\lambda$-model introduced in Lemma 41. Let $\mathbf{R}$ be the Cartesian product of all extensional reflexive Scott domains. Then we have $\operatorname{Ord}(\mathbf{P}) \subseteq \operatorname{Ord}(\mathbf{R})$. If $\operatorname{Ord}(\mathbf{R})$ is r.e., we get a contradiction as in the proof of Thm. 42.

\section{ACKNOWLEDGMENT}

Work partially supported by CONCERTO project.

\section{REFERENCES}

[1] H. P. Barendregt, The Lambda calculus: Its syntax and semantics, North-Holland, Amsterdam, 1984.

[2] H. P. Barendregt, M. Coppo, M. Dezani-Ciancaglini, A filter lambda model and the completeness of type assignment, Journal of Symbolic Logic 48 (4) (1983) 931-940.
[3] C. Berline, From computation to foundations via functions and application: The $\lambda$-calculus and its webbed models, TCS 249 (2000) 81-161.

[4] C. Berline, Graph models of $\lambda$-calculus at work, and variations, Math. Struct. in Comput. Sci. 16 (2006) 1-37.

[5] C. Berline, G. Manzonetto, A. Salibra, Lambda theories of effective lambda models, in: CSL'07, LNCS 4646, 2007.

[6] G. Berry, Stable models of typed lambda-calculi, in: ICALP'78, LNCS 62, Springer-Verlag, Berlin, 1978.

[7] A. Bucciarelli, T. Ehrhard, Sequentiality and strong stability, in: LICS'91, IEEE Computer Society Press, 1991.

[8] A. Bucciarelli, A. Salibra, Graph lambda theories, Math. Struct. in Comput. Sci. 18 (5) (2008) 975-1004.

[9] M. Coppo, M. Dezani-Ciancaglini, An extension of the basic functionality theory for the $\lambda$-calculus, Notre-Dame Journal of Formal Logic 21 (4) (1980) 685-693.

[10] M. Coppo, M. Dezani-Ciancaglini, F. Honsell, G. Longo, Extended type structures and filter lambda models, in: G. Lolli, G. Longo, A. Marcja (eds.), Logic Colloquium 82, NorthHolland, Amsterdam, the Netherlands, 1984.

[11] P. Di Gianantonio, F. Honsell, G. D. Plotkin, Uncountable limits and the lambda calculus, Nordic Journal of Computing 2 (2) (1995) 126-145.

[12] M. Droste, R. Gobel, Effectively given information systems and domains, in: CSL'89, 1989.

[13] F. Honsell, TLCA list of open problems: Problem 22, http://tlca.di.unito.it/opltlca/problem22.pdf (2007).

[14] F. Honsell, S. Ronchi Della Rocca, An approximation theorem for topological lambda models and the topological incompleteness of lambda calculus, Journal of Computer and System Sciences 45 (1992) 49-75.

[15] R. Kerth, Isomorphism and equational equivalence of continuous lambda models, Studia Logica 61 (1998) 403-415.

[16] R. Kerth, On the construction of stable models of $\lambda$-calculus, TCS 269 (2001) 23-46.

[17] G. D. Plotkin, Set-theoretical and other elementary models of the lambda-calculus, TCS 121 (1\&2) (1993) 351-409.

[18] A. Salibra, Topological incompleteness and order incompleteness of the lambda calculus, ACM Transactions on Computational Logic (4) (2003) 379-401.

[19] D. S. Scott, Continuous lattices, in: Toposes, algebraic geometry and logic, Springer-Verlag, Berlin, 1972.

[20] D. S. Scott, Domains for denotational semantics, in: ICALP '82, 1982.

[21] P. Selinger, Order-incompleteness and finite lambda reduction models, TCS 309 (2003) 43-63.

[22] V. Stoltenberg-Hansen, I. Lindstrom, E. R. Griffor, Mathematical theory of domains, Cambridge Univ. Press, 1994. 